\title{
Effect of particle shape on fluid statistics and particle dynamics in turbulent pipe flow ${ }^{\star}$
}

\author{
A. Gupta ${ }^{1, a}$, H.J.H. Clercx ${ }^{1}$, and F. Toschi ${ }^{1,2,3}$ \\ 1 Department of Applied Physics, Eindhoven University of Technology, Den Dolech 2, 5600 MB, Eindhoven, The Netherlands \\ 2 Istituto per le Applicazioni del Calcolo, Consiglio Nazionale delle Ricerche, Via dei Taurini 19, 00185, Roma, Italy \\ 3 Department of Mathematics and Computer Science, Eindhoven University of Technology, Den Dolech 2, 5600 MB, Eindhoven, \\ The Netherlands
}

Received 7 May 2018 and Received in final form 27 August 2018

Published online: 3 October 2018

(C) The Author(s) 2018. This article is published with open access at Springerlink.com

\begin{abstract}
Anisotropic particles are present in many natural and industrial flows. Here we perform direct numerical simulation (DNS) of turbulent pipe flows with dispersed finite-size prolate spheroids simulated by means of the lattice Boltzmann method (LBM). We consider three different particle shapes: spheroidal (aspect ratio 2 and 3) and spherical. These three simulations are complemented with a reference simulation of a single-phase flow. For the sake of comparison, all simulations, laden or unladen have the same energy input. The flow geometry used is a straight pipe with length eight times its radius where the fluid is randomly seeded with 256 finite-size particles. The volume fraction of particles in the flow has been kept fixed at $0.48 \%$ by varying the major and minor axis of each particle such that their volume remains the same. We studied the effect of different particle shapes on particle dynamics and orientation, as well as on the flow modulation. We show that the local accumulation of spheres close to the wall decreases for spheroids with increasing aspect ratio. These spheroidal particles rotate slower than spheres near to the wall and tend to stay with their major axes aligned to the flow streamwise direction. Despite the lower rotation rates, a higher intermittency in the rotational rates was observed for spheroids and this increase at increasing the aspect ratio. The drag reduction observed for particles with higher aspect ratio have also been investigated using the one-dimensional energy and dissipation spectra. These results point to the relevance of particle shapes on their dynamics and their influence on the turbulent flow.
\end{abstract}

\section{Introduction}

The study of solid particle suspensions in turbulence is relevant for the understanding and for the optimization of many biological and engineering flows [1]. Some examples of these are slurry flows, the combustion of pulverized solid fuels, dust storms, pollutant dispersion in the atmosphere and the dynamics and stresses on microalgae in photo-bioreactors. Additionally, non-spherical particles, with varying shapes and sizes are common: plankton species and pollen grains occur in an astonishing variety of shapes, cellulose fibers or textile vary in their rigidity and shape, microalgae in photo-bioreactors are also usually anisotropic in shape [2].

The motion of non-spherical particles in turbulent flows is particularly intricate, also in view of the fact that

\footnotetext{
* Contribution to the Topical Issue "Flowing Matter, Problems and Applications", edited by Federico Toschi, Ignacio Pagonabarraga Mora, Nuno Araujo, Marcello Sega.

a e-mail: a.gupta-1@tue.nl
}

their rotational dynamics in laminar flows is already complicated. The presence of turbulence makes the problem even more intriguing and challenging to solve: in addition to the complexity of turbulence, we now have to consider forces and torques that vary according to the particle orientation. Most of the literature on particulate fluid flows reports the fluid interactions with spherical particles [3-13]. Non-spherical particles, due to their anisotropic nature, are more complicated to treat than spherical particles. Consequently, relatively fewer numerical and experimental studies have been devoted to nonspherical particles in turbulent flows. For non-spherical particles, analytic approaches that account for fluid inertia in an unsteady flow and that are applicable across the full range of spheroidal shapes are not yet available in the literature [14].

The study of elongated particles suspended in a viscous fluid flow has been a topic for research through many decades. The case of an ellipsoidal particle immersed in a creeping viscous fluid was studied by Jeffery [15]. Further analytical work on particles of different geometrical shapes 
has been conducted by Brenner [16] and a general treatise is provided in Happel and Brenner [17]. All these different analytical studies assume Stokes flow conditions around the particles, i.e. viscous effects dominate over inertial effects.

A comprehensive review of the models used to describe non-spherical particle motion, along with numerical and experimental methods for measuring particle dynamics, has been provided in [14]. The majority of these previous studies, mostly focusing on turbulence, limited to the case of point-like spheroidal particles, assumed dilute conditions, and additionally, neglected the feedback on the flow. To the best of our knowledge, we can report only three investigations of finite-size anisotropic rigid particles in turbulence, those by Do-Quang et al. [18], Ardekani et al. [19] and Eshghinejadfard et al. [20]. The lattice Boltzmann method was used in [18] to simulate finite-size fibers in turbulent channel flows. In [19] researchers used an immersed boundary method to simulate oblate particles in a turbulent channel flow at volume fractions up to $20 \%$. Finally in [20], the lattice Boltzmann method coupled with an immersed boundary method was used to simulate fully resolved prolate ellipsoids with a different aspect ratio in a turbulent channel flow.

There are two important aspects regarding anisotropic particles in turbulence that we would like to explore further in this work. The first is the influence of particles on the carrier phase turbulence, commonly known as turbulence modulation. A fundamental understanding concerning the underlying phenomena, which are responsible for the complex interaction between the particles and turbulence is required to advance the design of engineering devices where such flows occur. The current understanding on the modification of the carrier phase turbulence due to the presence of solid particles, however, is limited. Different mechanisms have been suggested to explain experimental observations and different parameters have been identified as being important but a lack of consensus exists in this field. Moreover, it is difficult to separate the direct modulation of the turbulence which can be attributed to enhanced effective viscosity, from the additional effects, like the momentum exchange on the surface of particles, the presence of the particle wake or the distribution of particle-induced stresses.

For finite-sized particles, attenuation of turbulence of the carrier phase is observed with small particles while amplification is observed with larger particles [21,22]. The study of small spherical particles at higher concentrations was performed by Kulick and coworkers [23], modeling feedback on the flow (two-way coupling), reporting that the fluid turbulence is attenuated by the addition of particles, while turbulence anisotropy increases. This effect was reported to increase with the particle Stokes number, with the particle mass loading and with the distance from the wall. In the case of non-spherical particles, the shape effect of particles on the particle-turbulence interaction is even more interesting. It was first observed in 1948 that the addition of polymers to a turbulent pipe flow reduced the pressure drop substantially below that of the solvent alone at the same flow rate [24]. The phe- nomenon was termed as drag reduction and has been the subject of several numerical and experimental investigations since then. Compared to the rigid anisotropic particles, polymers have an additional degree of freedom: they can stretch. However, drag reduction in the suspensions of rigid fibers was also observed in [25, 26], similarly to what was reported for dilute polymer solutions [27, 28]. A review of the phenomenon of drag reduction was provided by Nieuwstadt and den Toonder [29]. While drag reduction was observed also with spherical particles [30], very few other evidences were reported in the literature. For example, an attenuation in turbulent kinetic energy of $3 \%$ and $15 \%$ respectively, for prolate ellipsoidal and for spherical particles, was observed [31]. In the maritime industry, drag reduction due to injection of air bubbles into the turbulent boundary layer under the ship hull is used to reduce the fuel consumption and has been a subject of several numerical and experimental studies (see review by Ceccio [32]). It has been observed that it dramatically depends on the bubble size and deformability [33]. Clearly, there is no general consensus on how drag reduction is affected by particle shape and particle properties. In this article, we will explore this further for rigid spheres and prolate spheroids.

Another important issue for anisotropic particles is their orientation and rotation. Particle orientation has a major influence on particle-fluid interactions. Orientation and angular velocities have been studied extensively in the past decade using the point-particle approach. Numerical and analytical studies on the orientation distribution of ellipsoids immersed in laminar and turbulent pipe flows have been performed [34], reporting that fibers in the laminar regime are more aligned in the flow direction. In several studies $[35,36]$, the deposition and orientation of glass fibers in a turbulent pipe flow was experimentally investigated. Researchers investigated, by means of DNS, the transport and the deposition of ellipsoidal particles in a turbulent channel flow [37]. They provided velocity and orientational particle statistics in the viscous sublayer and in the buffer layer. Later, it was observed that both spheres and ellipsoids are preferentially concentrated in the near-wall, low-speed streaks $[38,39]$. An investigation of the rotational motion of inertia-free spheroids in turbulent channel flow was conducted, see refs. [40,41]. These authors showed that oblate spheroids preferentially align their symmetry axes normal to the wall, whereas prolates are preferentially aligned parallel to the wall. The mean particle rotation was also reported to reduce when increasing the particle aspect ratio. In [42] researchers studied the effects of particle inertia, particle shape, and fluid shear on particle rotation using the direct numerical simulation of the turbulent channel flow. In [43], the work related to non-spherical particles in laminar shear flows and sedimentation of non-spherical particles in still fluids was studied.

In this article, we report results of simulations with particles with different shapes and try to understand their effect on the flow modulation, particle dynamics and orientation. The outline of the article is as follows. Section 2 deals with theory and introduces the approach used in 
this paper for particle-particle and particle-wall interaction. Section 3 comprises a discussion on flow geometry and particle shapes used for the simulations. Section 4 focusses on the fluid statistics where we present the mean and root-mean-square (RMS) velocity profiles along with the energy spectrum. In sect. 5, we discuss the results on particle dynamics. Here, the particle distribution profiles, velocity, and orientational statistics will be presented. Finally, sect. 6 summarizes and concludes the work.

\section{Methodology}

The simulation algorithm consists of three major components: the fluid solver, the model to simulate the dynamics of the particles and the particle-particle/particle-wall interaction.

The description of the lattice Boltzmann method as a flow solver has been discussed in several texts [44,45]. Lattice Boltzmann methods (LBM) are a class of computational fluid dynamics (CFD) methods for flow simulation. In LBM, instead of solving the Navier-Stokes equations, the discrete Boltzmann equation is solved to simulate the flow of a Newtonian fluid with collision models such as Bhatnagar-Gross-Krook (BGK). In this method, the fluid is replaced by distribution functions streaming along given directions and colliding at the lattice sites. The distribution function represents the probability of finding fictious fluid particles within a certain range of velocities and a certain range of locations at a given time. Both collision and streaming processes are local operations and therefore allows efficient programming for parallel computing. Additionally, the handling of complex phenomena such as moving boundaries, multiphase flows etc. can be handled in LBM without a need for a separate Lagrangian mesh. In comparison to molecular dynamics simulations, the distribution function replaces the tagging of each particle, thereby saving the computer resources drastically. By simulating streaming and collision processes across a limited number of distribution functions, the intrinsic particle interactions evolve the macroscopic behavior of the fluid.

The lattice Boltzmann equation reads as follows [44]:

$$
f_{i}\left(\vec{x}+\vec{e}_{i} \delta_{t}, t+\delta_{t}\right)=f_{i}(\vec{x}, t)+\frac{\delta_{t}}{\tau}\left(f_{i}^{e q}-f_{i}\right),
$$

where $f_{i}$ is the probability distribution function and the subscript $i$ labels a set of discrete speeds connecting the nodes of a regular lattice, $f_{i}^{e q}$ is the corresponding equilibrium distribution function, $\delta_{t}$ is the time increment and $\tau$ is the fluid relaxation time related to the kinematic viscosity as $\nu=\left(\tau-\delta_{t} / 2\right) c_{s}^{2}$, where $c_{s}$ is the speed of sound. The D3Q27 BGK lattice Boltzmann method has been used to simulate the fluid flow.

As also discussed in [13], the mid-point bounce-back method proposed by Chen et al. [46] has been used for the particle-fluid coupling. The net force and the torque on the suspended solid particles is computed using the momentum exchange with the surrounding fluid. Consequently,

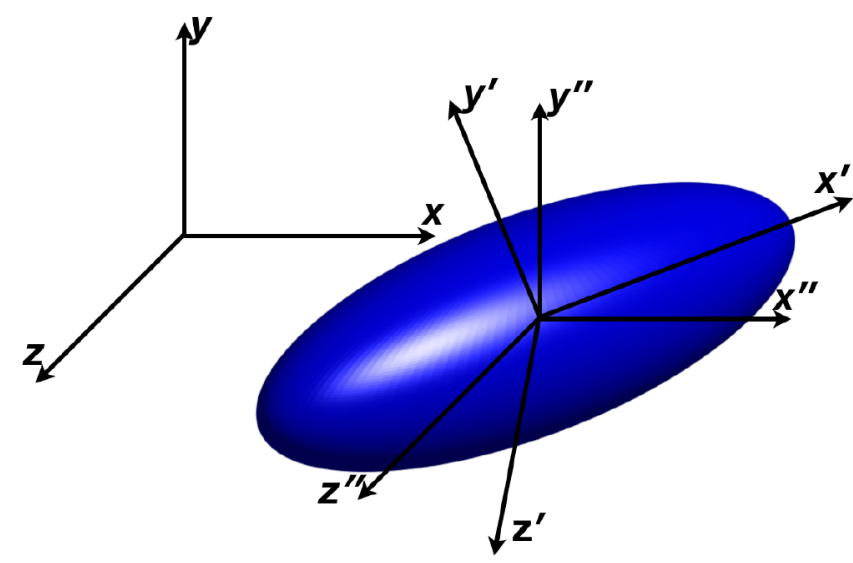

Fig. 1. An ellipsoidal particle and the corresponding coordinate systems: the inertial frame, $\mathbf{x}=(x, y, z)$, the particle frame, $\mathbf{x}^{\prime}=\left(x^{\prime}, y^{\prime}, z^{\prime}\right)$ and the comoving frame, $\mathbf{x}^{\prime \prime}=$ $\left(x^{\prime \prime}, y^{\prime \prime}, z^{\prime}\right)$.

the motion of the solid particle is simply determined by solving Newton's equations for the linear and angular momentum. Finally, these equations are integrated using a leap-frog scheme to obtain the complete motion of the suspended solid particles in the fluid [47].

In order to describe the motion of ellipsoids, we invoke three different Cartesian coordinate systems: the inertial frame, the particle frame, and the comoving frame. The inertial frame, $\mathbf{x}=(x, y, z)$, is the frame that spans the computational domain. The particle frame, $\mathbf{x}^{\prime}=\left(x^{\prime}, y^{\prime}, z^{\prime}\right)$, is attached to the particle with its origin at the particle mass center. In this frame, the coordinate axes are aligned with the principal directions of inertia. The comoving frame, $\mathbf{x}^{\prime \prime}=\left(x^{\prime \prime}, y^{\prime \prime}, z^{\prime \prime}\right)$, has its origin translating along the center of mass of the particle. The coordinate axes are parallel to those of the inertial frame. The different coordinate systems are shown in fig. 1 along with a particle of aspect ratio three.

The purpose of introducing the particle frame is to describe the orientational behavior of the ellipsoids using the principal of moments inertia (moment of inertia in the particle frame). The sole purpose of introducing the comoving system is to provide an intermediate step between conversion from inertial to particle frame. After the shift of origin from inertial to comoving frame, one needs to transform a given vector from the comoving frame to the particle fixed frame through the linear transformation $\mathbf{x}^{\prime}=\mathbf{A} \mathbf{x}^{\prime \prime}$. The orthogonal transformation matrix $\mathbf{A}$ comprises the nine direction cosines. Usually these parameters are comprised by the three independent Euler angles [47]. However, the Euler angles suffer from singularity problems. For this reason, the corresponding four Euler parameters will be used for the particle orientation. The parameters $q_{0}, q_{1}, q_{2}$, and $q_{3}$ are called quaternions. They are inter-dependent and must satisfy the following constraint: $q_{0}^{2}+q_{1}^{2}+q_{2}^{2}+q_{3}^{2}=1$. Using the quaternions, the transformation matrix is given by

$$
\mathbf{A}=\left(\begin{array}{lll}
a_{11} & a_{12} & a_{13} \\
a_{21} & a_{22} & a_{23} \\
a_{31} & a_{32} & a_{33}
\end{array}\right)
$$


Table 1. Parameter settings for the turbulence simulations (all dimensional quantities are in lattice units). $\langle g\rangle$ is the mean forcing needed to obtain fixed energy input, $u_{\tau}$ is the friction velocity, $t^{+}$is the integral time scale, $R e_{\tau}$ is the Reynolds number based on the friction velocity and on the diameter of the pipe $\left(R e_{\tau}=u_{\tau} D / \nu\right),\left\langle U_{x}\right\rangle$ is the average velocity in the pipe and $R e_{\left\langle U_{x}\right\rangle}$ is the Reynolds number based on average velocity and on the diameter of the pipe $\left(\operatorname{Re}_{\left\langle U_{x}\right\rangle}=\left\langle U_{x}\right\rangle D / \nu\right)$.

\begin{tabular}{|c|c|c|c|c|}
\hline Case & S0 & $\mathrm{S} 1$ & $\mathrm{~S} 2$ & S3 \\
\hline Aspect ratio & NA & 1 & 2 & 3 \\
\hline Major axis & NA & 5.8 & 9.16 & 12 \\
\hline Volume fractions & NA & $0.48 \%$ & $0.48 \%$ & $0.48 \%$ \\
\hline$\langle g\rangle$ & $2.06 \times 10^{-7}$ & $2.13 \times 10^{-7}$ & $2.11 \times 10^{-7}$ & $2.08 \times 10^{-7}$ \\
\hline$u_{\tau}$ & $3.5 \times 10^{-3}$ & $3.6 \times 10^{-3}$ & $3.5 \times 10^{-3}$ & $3.5 \times 10^{-3}$ \\
\hline$t^{+}$ & $3.39 \times 10^{4}$ & $3.34 \times 10^{4}$ & $3.35 \times 10^{4}$ & $3.38 \times 10^{4}$ \\
\hline$R e_{\tau}$ & 250 & 255 & 253 & 251 \\
\hline$\left\langle U_{x}\right\rangle$ & 0.0483 & 0.0468 & 0.0472 & 0.0478 \\
\hline$R e_{\left\langle U_{x}\right\rangle}$ & $3.45 \times 10^{3}$ & $3.34 \times 10^{3}$ & $3.37 \times 10^{3}$ & $3.39 \times 10^{3}$ \\
\hline
\end{tabular}

where $a_{i j}$ is

$$
\begin{aligned}
& a_{11}=q_{0}^{2}+q_{1}^{2}-q_{2}^{2}-q_{3}^{2}, \\
& a_{12}=2\left(q_{1} q_{2}+q_{0} q_{3}\right), \\
& a_{13}=2\left(q_{1} q_{3}-q_{0} q_{2}\right), \\
& a_{21}=2\left(q_{1} q_{2}-q_{0} q_{3}\right), \\
& a_{22}=q_{0}^{2}-q_{1}^{2}+q_{2}^{2}-q_{3}^{2}, \\
& a_{23}=2\left(q_{2} q_{3}+q_{0} q_{1}\right), \\
& a_{31}=2\left(q_{1} q_{3}+q_{0} q_{2}\right), \\
& a_{32}=2\left(q_{2} q_{3}-q_{0} q_{1}\right), \\
& a_{33}=q_{0}^{2}-q_{1}^{2}-q_{2}^{2}+q_{3}^{2} .
\end{aligned}
$$

The angular dynamics was integrated by the method of quaternions, as discussed in [47], for representing orientations and rotations of finite-size particles. The Euler equations are formulated in the particle frame and the principal moments of inertia are used.

The numerical method is capable of correctly predicting the particle dynamics as well as the velocity profiles of the turbulent flow in a pipe as discussed in [12]. However, the strong dependence of lubrication interactions on the minimum separation between two particles requires precise knowledge of the magnitude and direction of the minimum gap vector. For two spheres with given radii, this can be computed trivially using the distance between the centers and the radii of the particles. For spheroids, the problem is considerably more intricate. One solution is to follow the iterative procedure presented by Lin et al. [48] for the distance between two ellipsoids. The method involves the repositioning of tangent spheres along the inner surface of each ellipsoid to minimize the distance of the sphere centers and thus the gap between the ellipsoids. The approach is limited to ellipsoidal geometries and is not valid for particles of any arbitrary shape.

We developed a novel approach to compute the closest distance between the particles. In our approach, we randomly generate points constraint on the surface of the particles. The geometric minimum distance between two
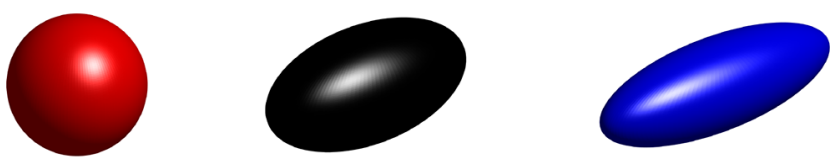

Fig. 2. A visual impression of the three particle shapes considered, $\mathrm{S} 1(\lambda=1), \mathrm{S} 2(\lambda=2), \mathrm{S} 3(\lambda=3)$. All particles have the same volume.

particles can then be computed as the minima in the distance function. In appendix A, we demonstrate the use of this method to calculate the distance between a sphere and a spheroid rotating in a Couette flow with an accuracy of about 0.5 lattice units. The method does not provide the direction of closest approach, and therefore, it is not possible to apply lubrication forces using this method.

\section{Flow geometry}

In this study, we performed a number of particle-laden DNS of turbulent pipe flow (see table 1 for details of simulations parameters). The flow geometry concerns a straight pipe and the fluid is randomly (with regard to position and orientation) seeded with 256 finite-size particles. Similar to most of the previous works in this field, we consider the case of axisymmetric ellipsoids, also called spheroids. Any spheroidal shape can be specified by a single aspect ratio, $\lambda$, defined as the ratio of the dimension along the symmetry axis to a perpendicular dimension: $\lambda=1$ corresponds to a sphere, $\lambda>1$ corresponds to a prolate spheroid (or fiber-like), and $\lambda<1$ corresponds to an oblate spheroid (or disk-like). In the present simulations, three different particle shapes are considered, either spheroidal (aspect ratio $\lambda=2$ and 3 ) or spherical $(\lambda=1$ ) as shown in fig. 2. The solid-phase volume fraction has been kept fixed at $0.48 \%$ by varying the major and minor axis of each particle such that its volume remains the same. Consequently, the volume fraction remains the same in all the particle-laden turbulence simulations considered 


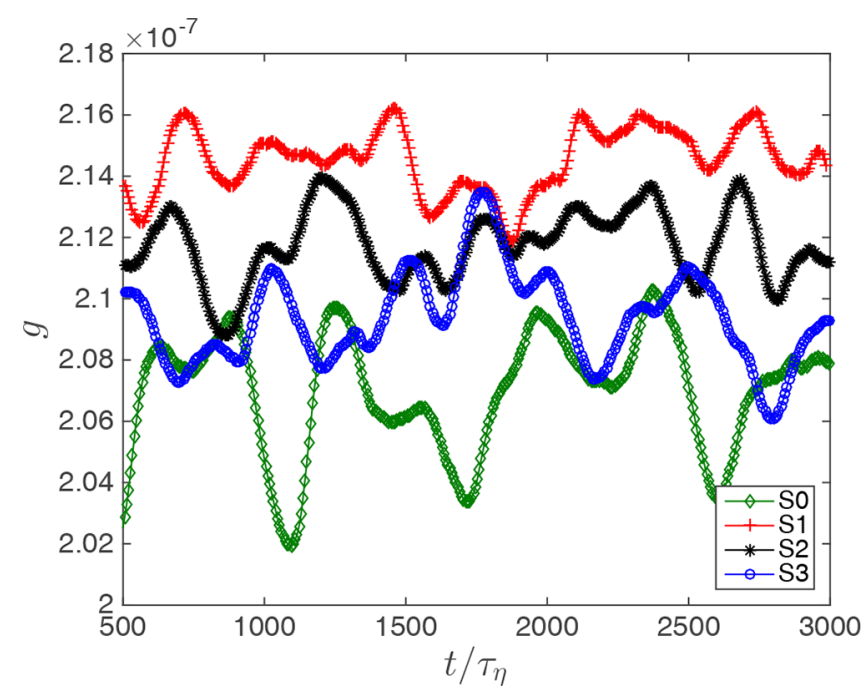

Fig. 3. Time history of the forcing signal $(g)$ for the three particle shapes considered, S1 $(\lambda=1), \mathrm{S} 2(\lambda=2), \mathrm{S} 3(\lambda=3)$ and for the unladen case, S0. The forcing $(g)$ is varied at each time step such that energy input remains constant, i.e. $E_{\text {in }}=$ $\langle\boldsymbol{g} \cdot \boldsymbol{U}\rangle_{1-\phi}=10^{-8}$. The time history is shown after the initial transient period $\left(t>500 \tau_{\eta}\right)$.

in this investigation. These simulations are complemented with a reference single-phase flow $(\mathrm{S} 0)$ : the unladen case with the same energy input as in the case with particles. Since the Cartesian coordinates were used for the simulations, the particle and fluid velocities were converted to radial coordinates before any further analysis.

As also discussed in $[12,13]$, the flow is characterized by a nearly constant energy input controlled by a time varying volume force, $\boldsymbol{g}=(g, 0,0)$, with intensity of $g$ chosen at each time step in order to produce a fixed energy input $E_{i n}=\langle\boldsymbol{g} \cdot \boldsymbol{U}\rangle_{1-\phi}=10^{-8}$. Here $\boldsymbol{U}=\left(U_{x}, U_{r}, U_{\theta}\right)$ is the velocity of the fluid, $\phi$ is the volume fraction of the particles and $\langle\cdot\rangle_{1-\phi}$ represents the fluid averaged dot product, i.e. the nodes within the particle boundaries are excluded from the average. The friction velocity is computed from the wall shear stress. The wall shear stress is computed from the momentum balance between pressure and viscous forces, using the relation, $\tau_{w}=\frac{1}{2} R \frac{\mathrm{d} p}{\mathrm{~d} x}$ with $\frac{\mathrm{d} p}{\mathrm{~d} x}=\langle g\rangle$, where $\langle g\rangle$ is the mean time-space averaged value volume force, $\boldsymbol{g}=(g, 0,0)$. The integral time scale is computed using the pipe radius and friction velocity $t^{+}=R / u_{\tau}$ and is shown in table 1.

The Reynolds number based on the friction velocity and on the diameter of the pipe $\left(R e_{\tau}=u_{\tau} D / \nu\right)$ is used in the subsequent discussion. The average velocity in the pipe, $\left\langle U_{x}\right\rangle$, and the Reynolds number based on it, $R e_{\left\langle U_{x}\right\rangle}=\left\langle U_{x}\right\rangle D / \nu$, are also presented in table 1 . The domain is discretized by a cubic mesh of $960 \times 240 \times 240$, with 960 lattice points in the streamwise and 240 in the lateral directions. The turbulent pipe flow is simulated via a circular duct element with length $L=8 R$, where $R$ is the pipe radius and periodic inlet/outlet boundary conditions. The rest of the details remains the same as discussed in $[12,13]$.
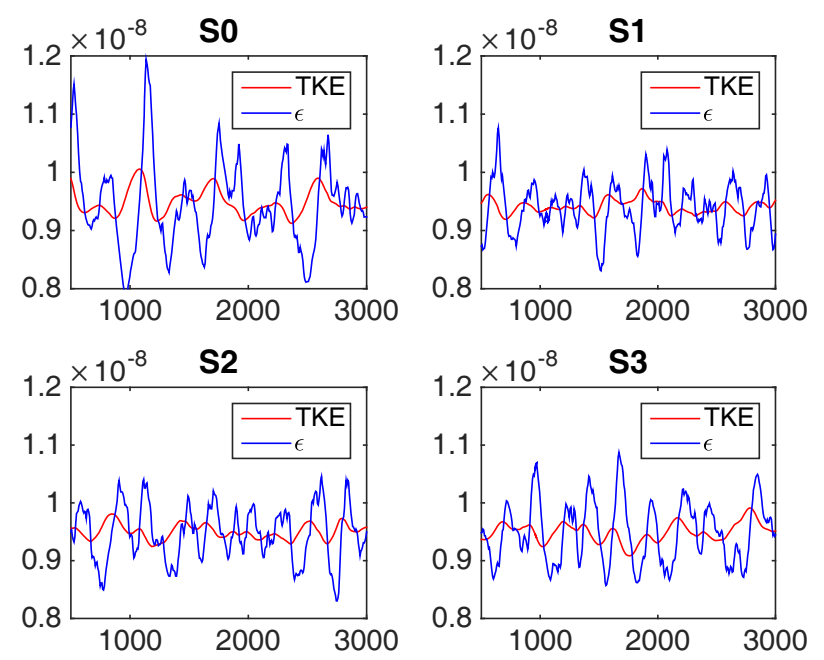

Fig. 4. Time series of the total kinetic energy $(T K E)$ and energy dissipation $(\epsilon)$ per unit length in turbulent pipe flow for single-phase flow (S0) and for the particle-laden turbulent pipe flow with three particle shapes, $\mathrm{S} 1(\lambda=1), \mathrm{S} 2(\lambda=2)$, S3 $(\lambda=3)$. The kinetic energy has been scaled down in the plot such that its RMS has the same value as the RMS of the energy dissipation. The time history is shown after the initial transient period $\left(t>500 \tau_{\eta}\right)$.

Figure 3 shows the variation of the forcing as a function of time (excluding the initial transient phase) for the three cases considered and S0. It can be observed that a larger forcing is needed for spherical particles $\mathrm{S} 1$, despite the lowest surface area. Table 1 also shows the mean forcing values, $\langle g\rangle$ needed for fixed energy input, which are used to compute the wall shear stress for each case.

Figure 4 shows the time history (excluding the initial transient phase) of the energy dissipation, $\epsilon$, and of the total kinetic energy ( $T K E$ ) per unit length. Here, a timelag between the two plots is suggestive of the presence of an energy cascade and thus of a turbulent flow. The kinetic energy has been scaled down in the plot such that its root-mean-square (RMS) value is the same as the RMS of the energy dissipation and the energy dissipation has been computed as discussed in [13].

\section{Fluid phase analysis}

A visual impression of the suspension flow in the whole domain is provided in fig. 5 , which shows a typical particle configuration along with a color plot of the instantaneous streamwise velocity. In the previous section, we observed that less forcing is needed to maintain the same energy input in turbulent pipe flow laden with spheroidal particles as compared to the case with spherical particles. In two-phase two-way coupled flow, several mechanisms contribute to the modification of the turbulence, e.g. increase of dissipation due to no-slip boundary conditions at the surface of the particles, the transfer of a part of the particle kinetic energy to the carrier phase and vice-versa. Secondary motion, due to the larger number of degrees 

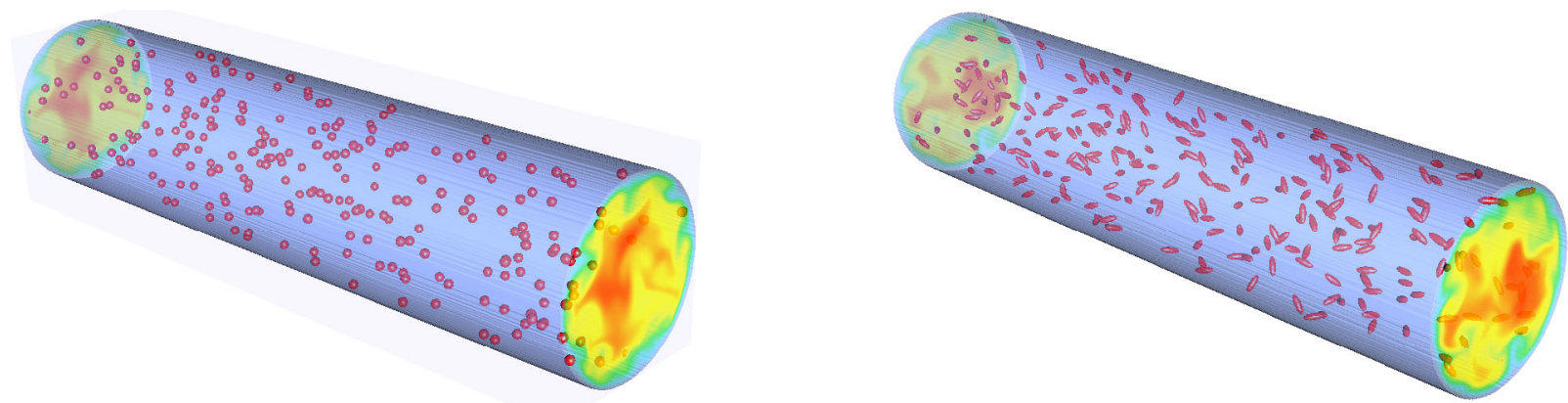

Fig. 5. Instantaneous snapshots of the streamwise velocity (color coded on vertical planes) together with the particle positions for the particle-laden turbulent pipe flow with particle shapes, S1 (spherical, left) and S3 (spheroidal, right). Color varies from blue (zero velocity near the wall) to red (maximum fluid velocity near the center of the pipe).

of freedom of motion (tumbling and spinning), associated with non-spherical particles can transfer kinetic energy of particles into turbulent kinetic energy, $E$, and transfer it in many more modes than spheres can do. Consequently, particles with different aspect ratio have different effect on the flow. In order to understand this, we decided to look into the energy and dissipation spectra for all the cases.

However, it is not straightforward to compute the spectra of the fluid in presence of finite-size particles. A simple way is to compute the spectra using the fluid velocity field at all the Eulerian mesh points in the computational domain including those points that are inside the particles at the time of interest. This means that the fluid motion inside the particle contributes to the computed spectrum of turbulent kinetic energy, $E$. The flow field inside the particle is the result of the rigid-body dynamics of the finite-sized particle. Of course, accounting for the fluid velocity inside the solid particles for computing $E(k)$ does not have any physical meaning. Moreover, setting the velocity inside the particle to zero only for computing $E(k)$ causes oscillations in the spectrum as discussed by Lucci and coworkers [49]. Their analysis suggests that including the Eulerian velocity field inside the particles to compute the energy spectrum not only has no physical meaning but also corrupts the spectrum at all the wave numbers. We therefore used an alternative approach to avoid the corruption of wave number spectra by the presence of particles in the Eulerian velocity field. We sample axial velocity fluctuations along the length of the pipe for every radial position. If during the sampling of the velocity signal a particle is encountered, that signal is rejected and not used for the computation of the spectrum. Therefore, the one-dimensional energy spectrum in the presence of finite-size particles can be written as

$$
E_{11}(k)= \begin{cases}\frac{1}{\pi} \int_{x=1}^{L} R_{11}(x) e^{-i k x} \mathrm{~d} x, & \text { if }(x=1, \ldots, L) \in f . \\ 0, & \text { otherwise }\end{cases}
$$

where $R_{11}(x)$ is the two-point velocity correlation for axial velocity fluctuations computed along the axial direction and $f$ represents the fluid nodes. Figure 6 shows the one-dimensional energy spectrum for turbulent fluc- tuations along the axial direction of the pipe for different particle shapes.

The energy spectra of the single-phase flow is also compared to the two-phase dispersed flows for all three particle shapes. The wave number is scaled by the Kolmogorov length scale and the energy is normalized using the energy dissipation and viscosity. The two vertical lines in the plot represent the wave numbers corresponding to the radius of the sphere, $\mathrm{S} 1$, and the semi-major axis of the most prolate particle, here S3. It can be observed from fig. 6 that the particles reduce the turbulent kinetic energy, $E(k)$, at small wave number compared to the case So. The decrease of kinetic energy at small wave numbers for spheroids is in agreement with results obtained from experimental studies [31]. This effect is more pronounced for spherical particles than for spheroidal particles (see inset of fig. 6). The area under the curve of the energy spectrum plot represents the kinetic energy due to turbulent fluctuations along the axial direction as shown in the equation below [50]:

$$
\overline{u_{x}^{\prime 2}}=\int_{k=0}^{k_{\max }} E_{11}(k) \mathrm{d} k
$$

The values $\overline{u_{x}^{\prime 2}}$ computed in this way, normalized by the value of $\overline{u_{x}^{\prime 2}}$ for the unladen case are $0.81,0.84$ and 0.95 for S1, S2 and S3, respectively. Furthermore, fig. 6 shows that particles increase the energy of the intermediate and high wave numbers. These observations can be qualitatively explained using the following phenomenological picture. In finite-size particle-laden turbulence, the large eddy structures have less energy than those in single-phase flow, because they are disrupted by the finite-size particles that drag the surrounding fluid in their direction. At the same time, particles generate, in their downstream direction, new eddies that become more frequent in the flow, thus increasing the energy of the high wave numbers. An increase of the kinetic energy at wave numbers corresponding to the particle size indicates that particles generate fluid motion at scales of the order of the particle size. Due to the relative motion between the particles and the fluid, velocity gradients are generated near the particle surface that enhance the rate of energy dissipation at large wave numbers and consequently suppress the kinetic energy in 

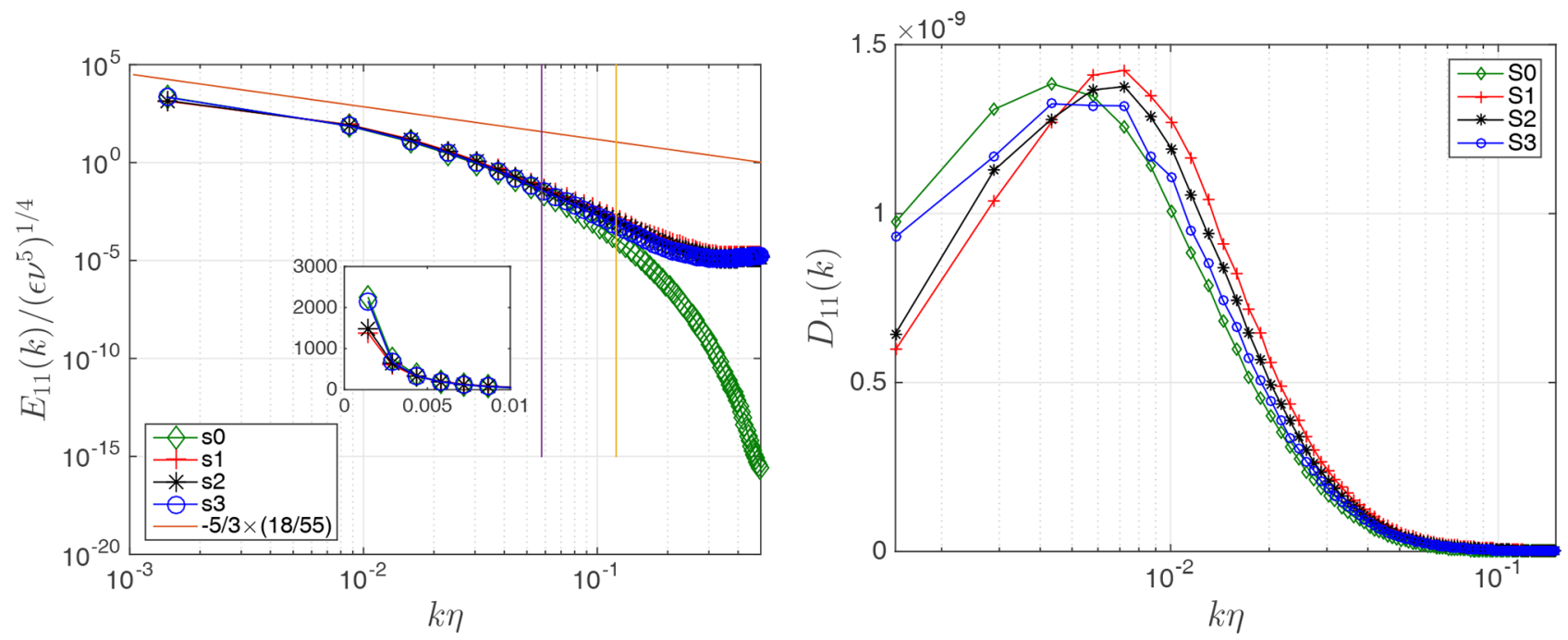

Fig. 6. One-dimensional energy spectra (left) and dissipation spectra (right) for turbulent fluctuations along the axial direction of the turbulent pipe flow, normalized by the energy dissipation and kinematic viscosity versus wave number normalized by the Kolmogorov length scale, $\eta$. The two vertical lines in the energy spectra plot represent the wave numbers corresponding to the radius of the sphere, $\mathrm{S} 1$, and the semi-major axis of the most prolate particle, here S3. A scaling exponent of $-5 / 3 \times$ $18 / 55$ corresponding the Kolmogorov hypotheses for the one-dimensional energy spectrum is also shown along with the energy spectrum [50].
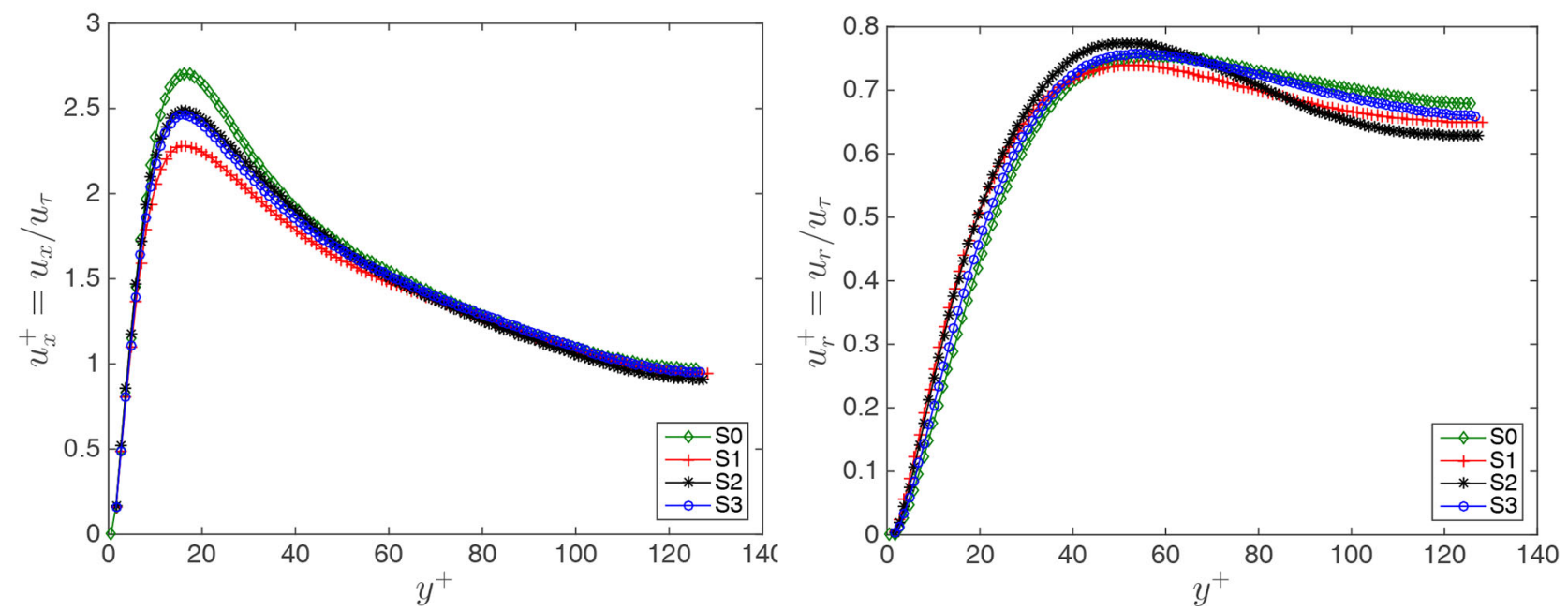

Fig. 7. The RMS of the axial, $u_{x}^{+}$, and of the radial fluctuating velocity component, $u_{r}^{+}$, for the fluid phase in viscous wall units for different particle shapes.

the spectrum at smaller wave numbers. To explore this further, we also looked into the one-dimensional dissipation spectrum. The one-dimensional dissipation spectrum can be computed from energy spectrum using [50]:

$$
D_{11}(k)=15 \nu k^{2} E_{11}(k) .
$$

It can be seen that the peak of the dissipation spectrum occurs at $k \eta \approx 0.006$. Thus the motion responsible for the bulk of dissipation is considerably larger than the Kolmogorov scale. It should be noted that there is no inconsistency between this observation and the Kolmogorov hypotheses. The hypotheses implies that the length scale of dissipative motions scales with $\eta$ and it does not necessarily have to be equal to $\eta$. The area under the curve of the dissipation spectrum plot represents the total dissipation due to turbulent fluctuations along the axial direction as shown in the equation below [50]:

$$
\epsilon=\int_{k=0}^{\infty} D_{11}(k) \mathrm{d} k .
$$

The values $\epsilon$ computed in this way, normalized by the value of $\epsilon$ for unladen case, are 1.24, 1.15 and 1.07 for S1, S2 and S3, respectively. This confirms that the spherical particles dissipate more energy than the other cases. In summary, spherical particles extract less energy at the smaller wave number and overall dissipates more energy. Overall, these combined effects can explain the higher drag and larger forcing for spherical particles. 


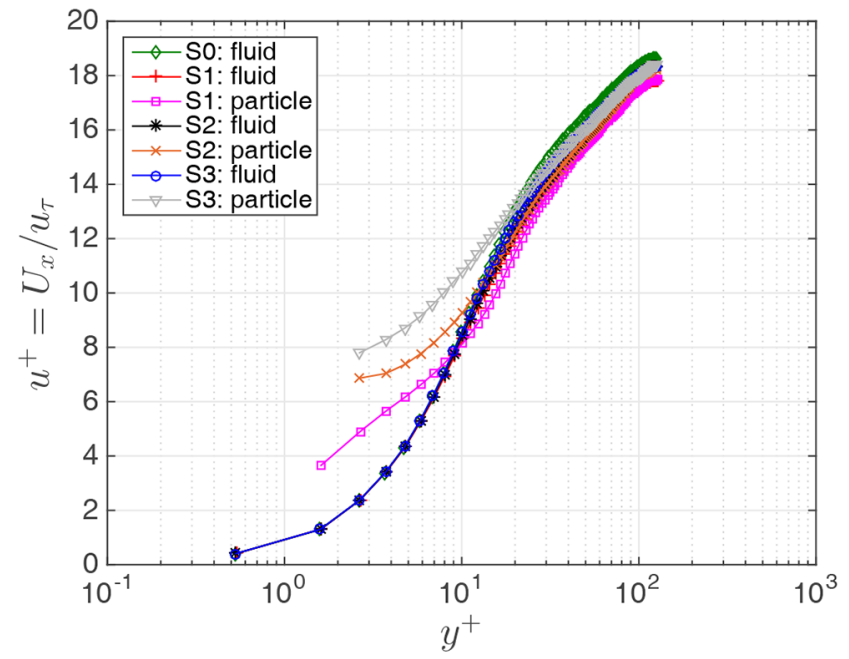

Fig. 8. Mean particle and fluid axial velocity profile $\left(U_{x}\right)$ in viscous wall units. The velocity statistics have been calculated using phase-ensemble average and rigid-body motion for lattice nodes inside the particle.

The spectrum is only for fluctuations in the axial direction. The particles also redistribute turbulent kinetic energy from axial to lateral directions. To understand this, we also discuss the effect of particles on the Eulerian root-mean-square (RMS) velocity profiles. The rootmean-square (RMS) velocity is normalized by the friction velocity, $u_{\tau}$. Figure 7 shows the axial and radial RMS velocity values for the three simulations.

Our results indicate that the presence of particles results in a decrease of the RMS of the streamwise velocity fluctuation and in an enhancement of the RMS of radial velocity fluctuations near to the wall. This behavior is consistent with the observation that the presence of particles redistributes energy more isotropically $[8,13]$. However, a decrease in streamwise velocity fluctuations in the presence of spherical particles has also been observed for a turbulent pipe [4]. But they also used a smaller pipe to particle diameter ratio of 15 as compared to our simulations (almost 20).

It is also observed that the effect is less pronounced for particles with higher aspect ratio for streamwise velocity fluctuations, i.e. the streamwise velocity fluctuation are less dampened by the presence of particles with higher aspect ratio. This is consistent with the observation that among the particle-laden cases, spheroidal particles with higher aspect ratio have higher turbulence kinetic energy at low wave numbers in the energy spectrum. With respect to the radial RMS fluctuations, it is observed that the profiles for higher aspect ratio spheroid (S3) particles are closer to a single-phase flow along most of the pipe radius.

Figure 8 depicts the mean particle and fluid velocity profiles in viscous wall units. The fluid velocity statistics have been calculated using phase-ensemble averaging as discussed in [13]. Similarly, the particle velocity profile is computed using the rigid-body motion for lattice nodes inside the particle. It can be observed in fig. 8 that both fluid and particles have the same mean velocity in the whole

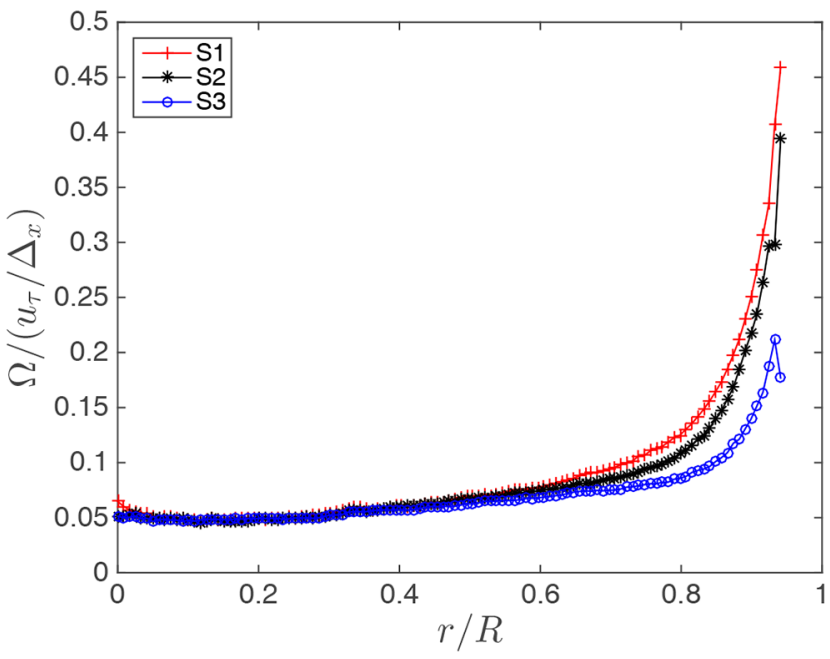

Fig. 9. Mean absolute value of the particle angular velocities versus the distance from the center of the pipe to the wall, normalized with the friction velocity, for the three particle shapes, $\mathrm{S} 1(\lambda=1), \mathrm{S} 2(\lambda=2), \mathrm{S} 3(\lambda=3)$. The error bars computed using the RMS of the corresponding angular velocities were at least three orders of magnitude smaller than the mean values.

pipe with the exception of velocities near the wall where particles have a larger mean velocity than the surrounding fluid. For the particles, the velocity at the wall does not have to be zero as the particles can have a relative tangential motion. Since particles have finite size, they might be located in regions with a higher velocity. This results in higher average velocity of particles compared to local fluid layers. Moreover, spheroids moves faster than spheres in the near-wall region. It is later shown that spheroids tend to align themselves in streamwise direction. This streamwise alignment might be the reason for higher velocity of spheroids compared to spheres.

\section{Particle dynamics}

Anisotropic particles in turbulence exhibit rich orientational dynamics from the coupling of their rotation to the velocity gradients of the turbulence field. We consider the rotation and orientation of neutrally buoyant axisymmetric spheroidal particles suspended in a turbulent pipe flow. Using numerical calculations, we explore how particle rotation depends upon particle shape. In the subsequent discussion, $r$ is the radial coordinate in the cylindrical coordinate system with the pipe center as the origin and $R$ being the radius of the pipe. Note that this radial coordinate system is different from the Cartesian coordinate system discussed earlier in sect. 2 and used solely for the presentation of results. While computing the radial averages, the radial position of the centre of mass of a particle is used. This means that all data points with centre of mass of a particle lying between any given integer radial position, $r_{o}-1$ and the next integer position, $r_{o}$, are averaged together and returns the mean value at $r_{o}$. For a given quantity $q$, whose radial average is being computed, 

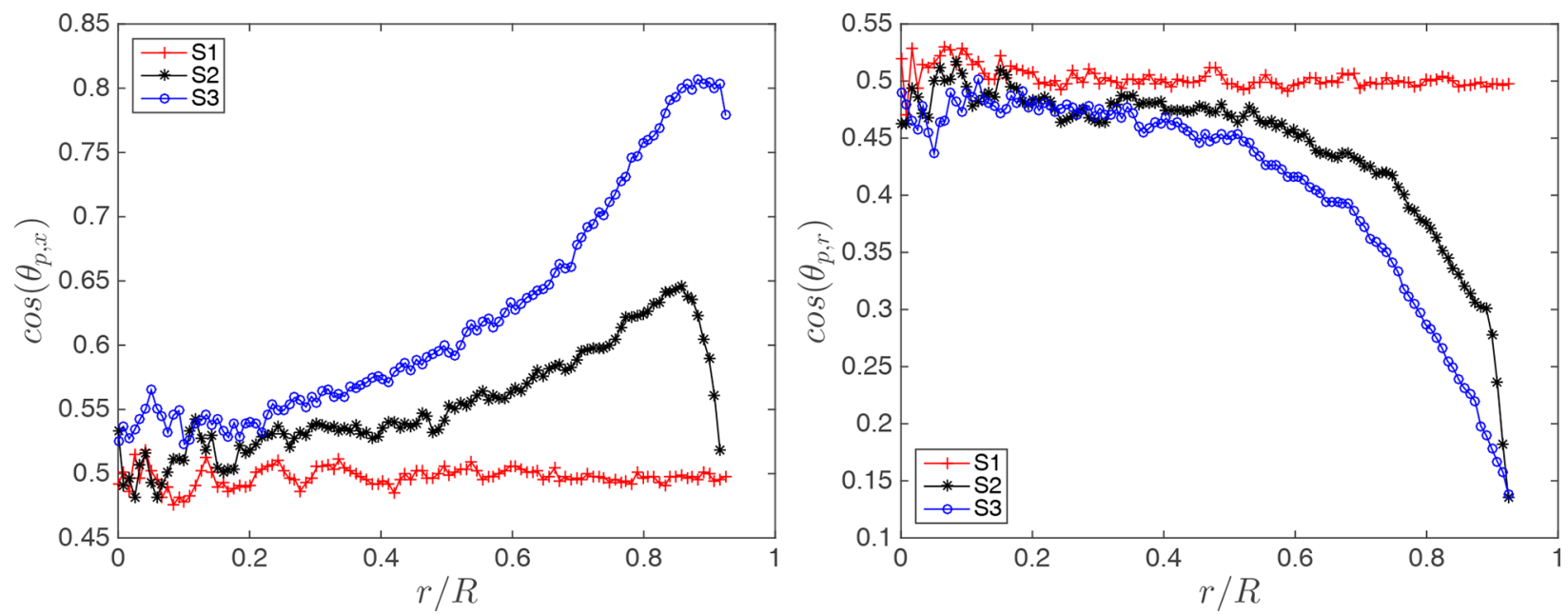

Fig. 10. The absolute mean direction cosine of the particle inclination angle, measured with respect to the axial (left panel) and radial directions (right panel), as a function of the distance from the center of the pipe to the wall, corresponding to the three particle shapes, $\mathrm{S} 1(\lambda=1), \mathrm{S} 2(\lambda=2), \mathrm{S} 3(\lambda=3)$.

this can be written as follows:

$$
q\left(r_{o}\right)=\left\langle q(r) \mid\left(r_{o}-1\right)<r \leq r_{o}\right\rangle
$$

where $r_{o}$ is the integer radial coordinate and $r$ is the position of centre of mass of the particle.

Inertial particles do not just sample fluid vorticity and strain, but rather they extract angular momentum, transport it, and return it back to the fluid phase. This depends upon how the total angular velocity of the particle is distributed between tumbling and spinning. This distribution of total angular velocity between tumbling and spinning is likely to affect these phenomena. The tumbling denotes the rotation of the symmetry axis of the particle, while the term spinning denotes the rotation of the particle around its own symmetry axis. An axisymmetric particle with symmetry axis given by the unit vector $\mathbf{p}$ rotates with a solid-body rotation rate, $\Omega=\left(\Omega_{x}, \Omega_{r}, \Omega_{\theta}\right)$, that can be split into a spinning rate which can be computed as the dot product of the angular velocity, $\boldsymbol{\Omega}$, with the unit vector along the symmetry axis, $\mathbf{p}$ :

$$
\Omega_{s}=|\boldsymbol{\Omega} \cdot \mathbf{p}|,
$$

and a tumbling rate, which is the cross product of the angular velocity, $\boldsymbol{\Omega}$, with the unit vector along the symmetry axis, p:

$$
\Omega_{t}=|\mathbf{p} \times(\boldsymbol{\Omega} \times \mathbf{p})| .
$$

The mean absolute value of the particle angular velocities, $|\boldsymbol{\Omega}|$ as a function of radial positions, is reported in fig. 9 . The rotation velocities are quite similar far from the wall for all the particle shapes. It can be observed that the prolate particles have significantly lower rotation rates than spheres close to the wall. The mean particle rotation was also reported to reduce close to the wall when increasing the particle aspect ratio [19,20]. Even a small drop in the angular velocity is observed near the wall for the largest aspect ratio case, S3. This might be due the physical interference of the wall with rotating motion of the particles which becomes more significant on increasing aspect ratio.
Figure 10 depicts the absolute mean direction cosines, $\langle|\cos (\theta, i)|\rangle$, versus distance from the center of the pipe for the axial (left) and radial (right) directions, where $(\theta, i)$ is the angle between the symmetry axis of the spheroid and the relevant direction of the inertial reference frame. Note that values of $\cos \left(\theta_{p, x}\right)$ close to 1 indicate that the particles tend to be aligned with their symmetry axis parallel to the axial direction. It can be immediately seen that finitesize spheroids tend to be aligned with their symmetry axis along the streamwise direction, in particular close to the wall, where particles exhibit very strong preferential orientation. This can be observed by an increase in $\cos \left(\theta_{p, x}\right)$ and decrease of $\cos \left(\theta_{p, r}\right)$ near to the wall. The effect of particle shape on both axial and radial direction cosines increases with increase in particle aspect ratio. This preferential orientation decreases gradually toward the pipe center. The minor fluctuations appearing on the curves (in particular near the pipe center) are due to the discrete nature of the solid particles and to the limited amount of particles available for statistics. In the core region, the orientations are more isotropic and random orientation is thus more probable, which is due to higher isotropy of the flow field fluctuations around the pipe center. Consequently, both the axial and radial direction cosines for all the particle shapes approach 0.5 and accordingly reflect an isotropic orientation in the core region of the pipe. Obviously, the orientation of a sphere always remains random as it does not have any preferred axis. Alignment of finite-size spheroids in our study is qualitatively similar to the results obtained for inertial point particles as reported in $[38,39,42]$ and for finite-size particles as reported in [20] for turbulent channel flow. Spheres does not have one particular symmetry axis. The orientation data was plotted for sphere as a sanity check and the symmetry axis was fixed arbitrary for spheres in our analysis.

Figure 11 shows the mean spinning and tumbling angular velocities versus the distance from the center of the pipe to the wall, for different particle shapes, normal- 

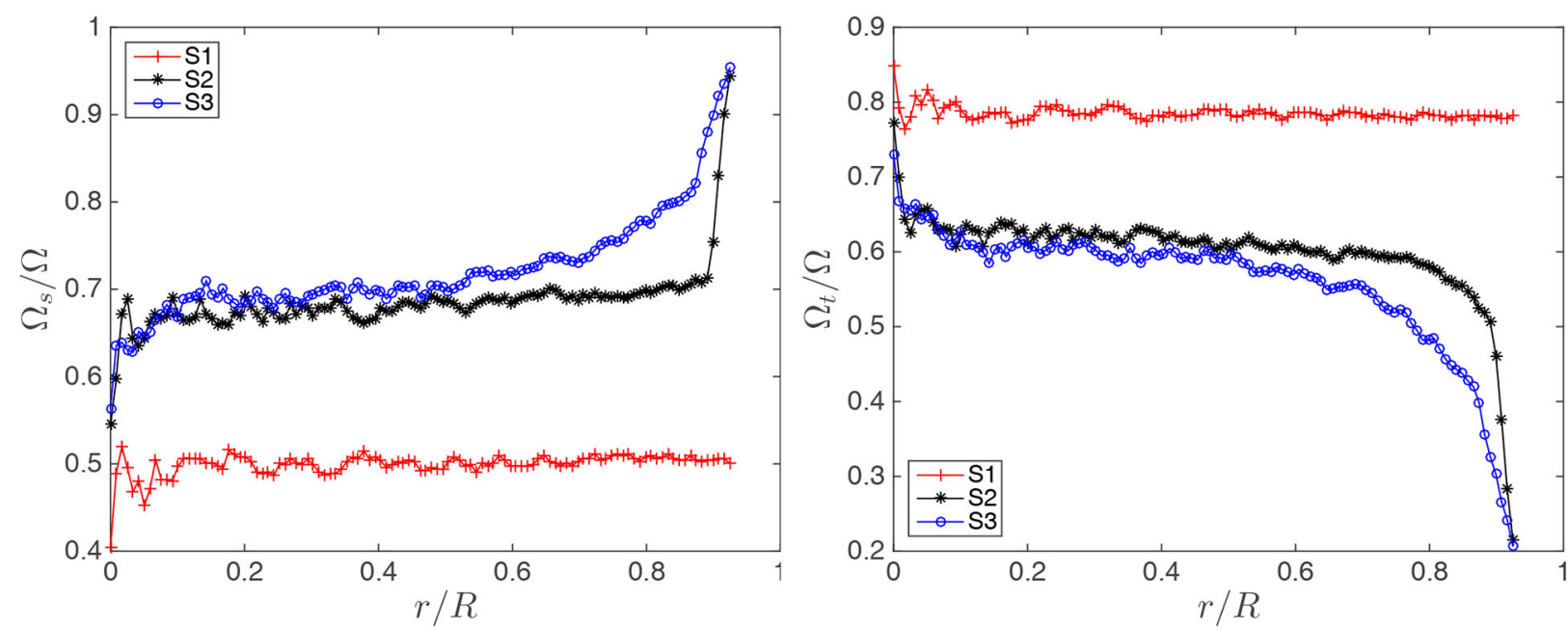

Fig. 11. Mean spinning, $\Omega_{s}=\boldsymbol{\Omega} \cdot \mathbf{p}$ and tumbling angular velocities, $\Omega_{\boldsymbol{t}}=|\mathbf{p} \times(\boldsymbol{\Omega} \times \mathbf{p})|$ versus the distance from the center of the pipe to the wall, for the three particle shapes, S1 $(\lambda=1)$, S2 $(\lambda=2)$, S3 $(\lambda=3)$, normalized with the mean absolute value of the particle angular velocities.

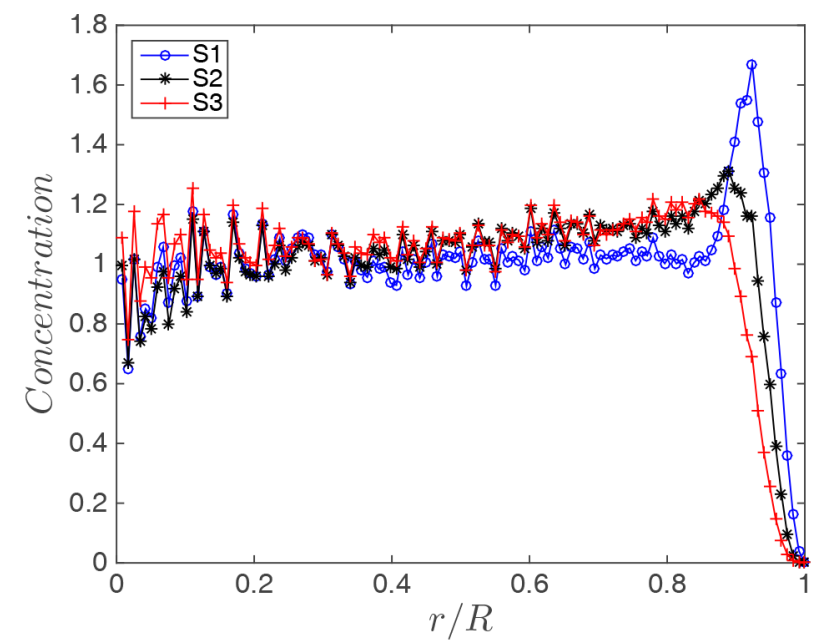

Fig. 12. The particle concentration profile across the pipe radius for the three particle shapes, $\mathrm{S} 1(\lambda=1), \mathrm{S} 2(\lambda=2), \mathrm{S} 3$ $(\lambda=3)$. The profiles have been normalized such that the area under the curve remains one. The large fluctuations near the center of the pipe stems from the limited amount number of particles available for statistics due to less cross-sectional area near the center of the pipe.

ized with the mean absolute value of the particle angular velocities. Spheroids align parallel to the axial direction near to the wall. This results in a higher relative spinning rate for spheroids as we go towards the wall. This alignment and the corresponding spinning effect becomes slightly stronger for particles with greater departure from sphericity. A complementary behavior is observed for the tumbling profiles, where the tumbling decreases as the non-spherical particle approaches the wall. As expected, the radial profiles of tumbling and spinning velocities for spheres are independent of the radial position.
The local volume fraction of particles for different particle shapes is shown in fig. 12. The concentration profiles have been normalized such that the area under the curve remains one. It can be seen that spherical particles have a local peak of distribution close to the wall. For spheroids, the peak seems to diminish with increasing aspect ratio such that there is no clear local peak for S3. This means that spheroidal particles apparently do not show preferential concentration near to the wall. A similar phenomenon was observed in an experimental study of fiber suspensions by [51] and in a numerical study of spheroids [52]. On the other hand, spherical particles stay mainly trapped close to the wall. The main factor that helps spheroids leaving this region can be their increased collision probability with the wall due to their anisotropy. Far from the wall, where the mean shear vanishes, this preferential concentration disappears and the behavior is similar to that observed in homogeneous and isotropic turbulence.

Figure 13 shows a numerically measured trajectory of a spheroid with $\lambda=3$. This example illustrates an important property of the rotation of particles: intermittency. The particle has bursts of high tumbling rate with smooth rotational behavior in between the bursts, reflecting the intermittency of particle rotations. To understand this rotational intermittency further, we plot the probability density function ( $p d f)$ of tumbling rate squared for spheroids and spheres together with the $p d f$ of enstrophy (vorticity squared) for the unladen case in fig. 14. The values are normalized with their respective means.

Spheres simply tumble with the vorticity for lower rotational rates. Variations in the orientation of the spheroids with respect to the velocity gradient tensor contribute additional fluctuations giving spheroids the most high rotation rate events that increases with the aspect ratio. The results indicates a similar trend as observed in [53] for homogeneous isotropic turbulence.

Figure 15 and 16 show the results for the joint probability distribution function (PDF) of axial and angular 


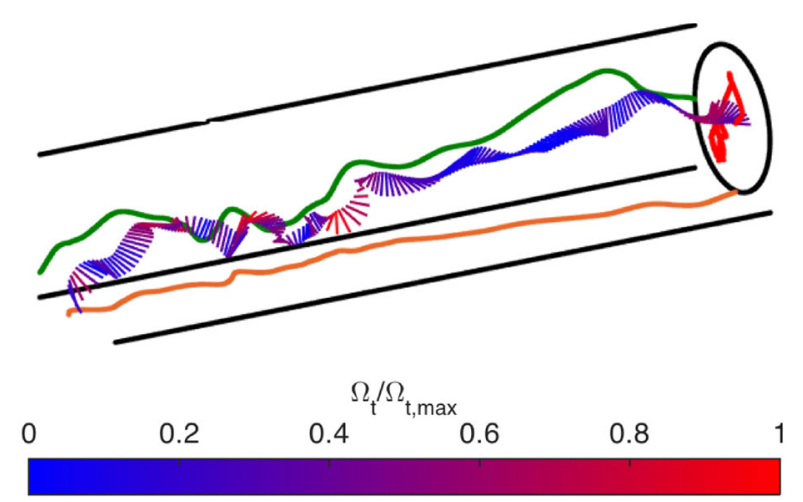

Fig. 13. Three-dimensional view of a spheroid trajectory (S3) in the turbulent pipe flow for 10 eddy turnover times with its projection on three orthogonal planes. The color code of the rod represents the rotation rate. The black line indicates the projection of boundaries of the pipe on the orthogonal planes. For the purpose of visualization, the axial direction has been squeezed by a factor of 20 .

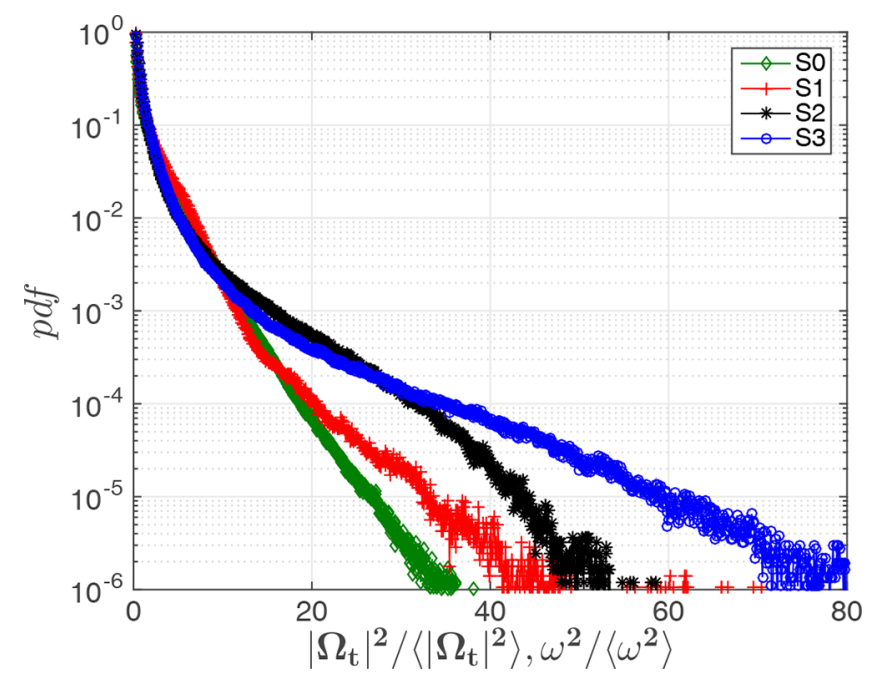

Fig. 14. Probability density function $(p d f)$ of the tumbling rate squared for spheroids and spheres, $\left|\boldsymbol{\Omega}_{\boldsymbol{t}}\right|^{2}$, normalized with respect to their mean, $\left\langle\left|\Omega_{t}\right|^{2}\right\rangle$, for the three particle shapes, S1 $(\lambda=1), \mathrm{S} 2(\lambda=2), \mathrm{S} 3(\lambda=3)$, compared with the probability density function of enstrophy for the unladen case $(\mathrm{S} 0), \omega^{2}$, normalized with respect to its mean value, $\left\langle\omega^{2}\right\rangle$.

velocity, respectively, with the radial position of the center of mass of the particles obtained from the simulation. The axial velocity is normalized by the friction velocity of the corresponding simulation, the angular velocity is normalized by the ratio of friction velocity to lattice resolution $\left(\Delta_{x}=1\right)$ and the radial positions are normalized with the radius of the pipe.

The particle axial velocity is compared with the mean Eulerian velocity obtained from another simulation without particles but with the same energy input. It is observed that the axial velocity of particles is scattered around the mean velocity obtained from the Eulerian field. The angular velocity of a sphere in a shear flow with shear rate $G$ is given by $G / 2$. For this reason, the magnitude of angular velocities of the particles is compared with the half of the shear rate $(G / 2)$, again obtained from another simulation with the same energy input but without particles. The angular velocities of the particles increase towards the wall due to the presence of a high-shear region and remains scattered around the values of shear rate obtained from the Eulerian field. The finite size of the particles results in an excluded region near to the wall. Changing the particle aspect ratio has a more prominent effect on angular dynamics than on the center-of-mass dynamics. There are few observations that can be made by looking at angular velocities. First, S3 samples angular velocities are very close to zero near to the wall while the sphere S1 only exhibits angular velocities greater than zero. This can be explained by the fact that the spheroids have higher spinning rate near to the wall than the tumbling rate which results in lower angular velocities overall. It can be seen that the maximum values of angular velocities encountered decreases on increasing the aspect ratio, which is consistent with the observation made in fig. 9. The axial velocity joint $\mathrm{PDF}$ does not change much at changing the particle shapes, apart from the particle velocity near the wall, which remains higher for higher aspect ratio particles as also observed in fig. 8 .

\section{Conclusions}

In this paper, the Lattice Boltzmann method (LBM) has been used to simulate turbulent pipe flow seeded with finite-size prolate spheroids and spheres. Test cases with spheroidal particles of aspect ratio two and three, along with a case with spherical particles and an unladen case have been successfully investigated. Investigations of finite-size anisotropic particles in turbulence are scarce and the DNS of fully resolved prolate spheroids in turbulent pipe flow has not been performed yet to our knowledge. Therefore, it is not possible to make a quantitative comparison with the existing literature. The outcome of our analysis is a rather qualitative description of particle dynamics and its effect on flow statistics in a turbulent pipe flow for spheroidal particles with different aspect ratios.

Comparing the obtained results of simulations with spheroidal particles with those of spherical particles, it is found that finite-size spheroids experience considerably lower angular velocities close to the wall and stay prevalently aligned parallel to the wall. Despite the lower rotation rates, the variations in the orientation of the spheroids with respect to the velocity gradient tensor results in higher rotational intermittency, which increases with increasing aspect ratio. A mean particle velocity that is higher than the mean fluid velocity near to the wall was also observed. Streamwise turbulence attenuation occurs for all particle types. However, the effect is more pronounced for spherical ones. For radial and tangential directions, turbulence fluctuations for spheroids are closer to single-phase flow. Although spheres show a local peak of volume fraction near the wall, this is clearly not the case for spheroids. Spheroids are mainly aligned along 

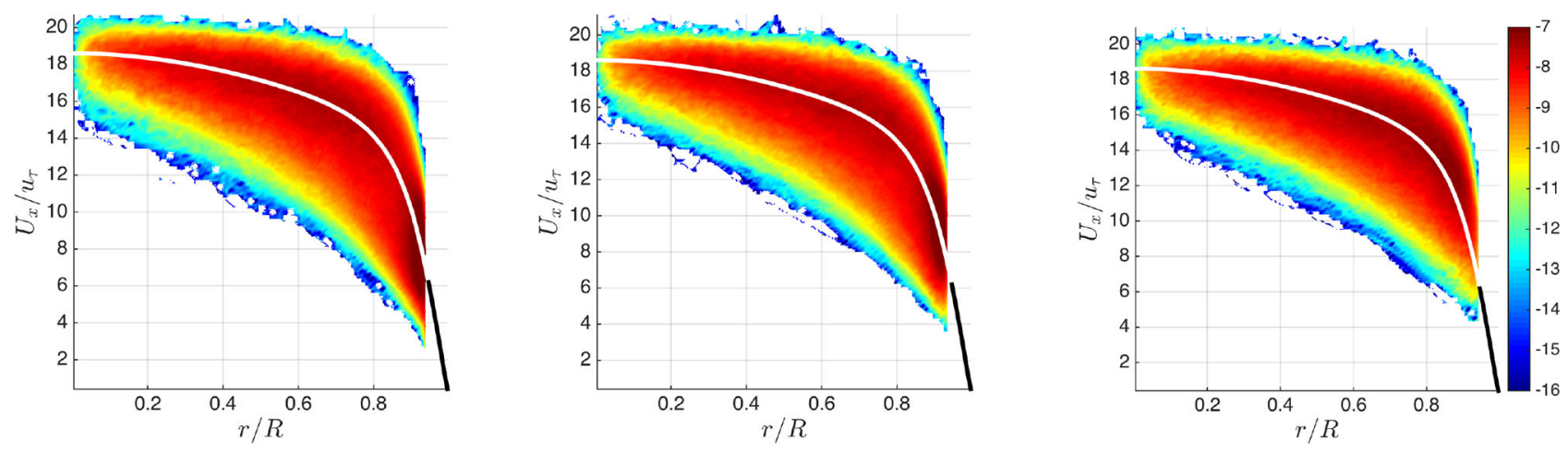

Fig. 15. Joint PDF of the axial velocity with radial position of the particles for the three particle shapes, $\mathrm{S} 1$ ( $\lambda=1$, left), $\mathrm{S} 2\left(\lambda=2\right.$, centre), S3 $\left(\lambda=3\right.$, right). Color bar indicates $\log _{10}$ of probability. White line inside colors and black line outside indicate the mean axial velocity obtained from the Eulerian velocity field of the turbulent pipe flow simulation without particles and with the same energy input as the one with particles (S0).
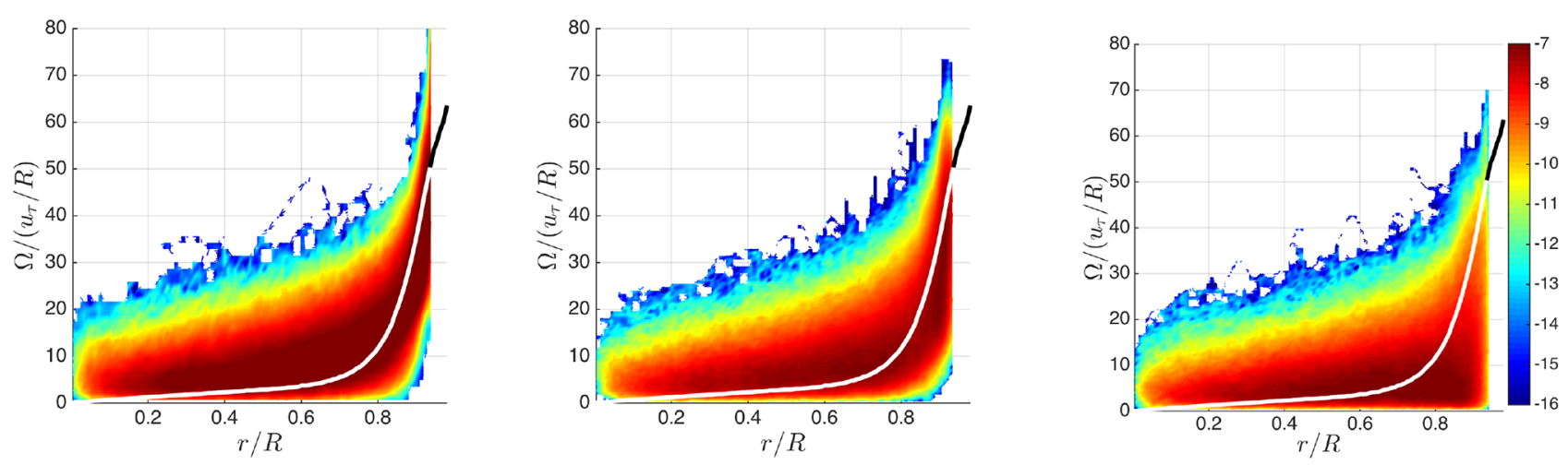

Fig. 16. Joint PDF of the magnitude of the angular velocity with radial position of the particles for the three particle shapes, S1 $(\lambda=1$, left $), \mathrm{S} 2\left(\lambda=2\right.$, centre), S3 $\left(\lambda=3\right.$, right). The color bar indicates $\log _{10}$ of probability. The white line inside colors and the black line outside indicate half of the mean shear rate obtained from the Eulerian velocity field of the turbulent pipe flow simulation without particles and with the same energy input as the one with particles (S0).

the streamwise direction. The preferential orientation and spinning are stronger close to the walls and increase with the particle aspect ratio. Around the pipe center, particles show a much less preferential orientation. Another macroscopic effect of non-spherical particle dynamics includes drag reduction compared to the spherical particles. The results show that the turbulent kinetic energy decreases and dissipation increases in the presence of spherical particles as compared to the spheroidal particles. The energy present in fluid-phase velocity fluctuations is shifted to smaller scales by the presence of particles. These observations were used to explain the higher forcing needed for spherical particles.

The results presented in this article are qualitatively in agreement with those of Eshghinejadfard et al. [20], where they simulated finite-size prolate spheroids in a turbulent channel. They also observed stronger streamwise turbulence attenuation for spherical particles along with a peak in volume fraction, that disappears for particles with increasing aspect ratio. Moreover, they also observed a preferential alignment of spheroids with axial direction which increases with particle aspect ratio and is stronger close to the wall. The effect of particle shape on turbulent kinetic energy was qualitatively similar to that observed by Bellani et al. [31] where they observed that addition of both spherical and spheroidal particles results in attenuation of turbulent kinetic energy with more pronounced effect of spheroidal particles.

As a continuation of the present work, it might be interesting to study if the turbulence is attenuated or enhanced on varying pipe to particle diameter ratio. Furthermore, a momentum budget analysis can be useful as well to understand the relative contribution of Reynolds shear stress and the particle-induced stresses.

Though the method for particle-particle interaction presented in this paper works for particles with any given shape, it has the disadvantage that it cannot be used to implement the lubrication forces. Four-way coupled numerical simulation algorithms capable of handling arbitrarily shaped particles in turbulence are needed. Another limitation of the work presented here is the limited parameter space that is explored: large portions of the parameter space is yet to be explored and many aspects of the complex cases relevant to applications still need to be understood. However, thanks for systematic investigations, it will be possible to provide a physical explanation to the 
mechanism of particle-induced turbulent drag reduction and enhance the general understanding about particles dynamics in turbulence.

This work is part of the Industrial Partnership Programme (IPP) Computational Sciences for Energy Research of the (former) Foundation for Fundamental Research on Matter (FOM), which is (now) part of the Netherlands Organization for Scientific Research (NWO). This research programme is co-financed by Shell Global Solutions International B.V. The authors gratefully acknowledge the support of the NWO for the use of supercomputer facilities (Cartesius) under Grant No. SH-33415. Finally, the authors would like to thank their colleagues Gianluca Di Staso and Pinaki Kumar from TU Eindhoven for the numerous scientific discussions that greatly helped to improve the quality of the manuscript and to Xiao Xue for improving the particle update algorithm.

\section{Author contribution statement}

AG, FT and HC devised the idea of the work. AG adapted the necessary code, performed the numerical simulations and performed the data analysis. All authors discussed the results and contributed to the final manuscript. AG drafted the first version of the manuscript with support from FT and HC. All the authors have read and approved the final manuscript.

\section{Appendix A. Model for spheroid interaction}

We used a very simplistic model for the particle-particle and particle-wall interaction. In our model, if a particle hits the wall or another particle, it is elastically bounced off affecting only the component of the velocities that are directed along the contact normal and leaving the tangential components and angular momentum unaffected. In order to use this approach, we just need the geometric (minimum) distance between two arbitrary surfaces and not the gap vector. However, the problem of finding the minimum distance between two arbitrary surfaces is also rather difficult. We implemented an approximate technique based on the Monte Carlo method to estimate the minimum distance between the two particles or the particles and the wall. It is based on repeatedly finding the distance between randomly generated points on the surface of the particles, which can be done analytically and then find the minima/maxima of the distance. In fig. 17, we employ this method to compute the distance between two stationary spheroids as a function of the number of randomly generated points on the surface of each spheroid, $N_{p}$. The actual minimum distance between the objects is 4 lattice units and different colors represent different numerical experiments. It can be observed that the algorithm converges to the actual separation between the particles with an accuracy of 0.5 lattice units for $10^{4}$ iterations.

For computing the minimum distance between two particles $i$ and $j$, we generate $n$ points on the surface of each particle, $\mathbf{r}_{i, n}=\left(x_{i, n}, y_{i, n}, z_{i, n}\right)$ and $\mathbf{r}_{j, n}=$

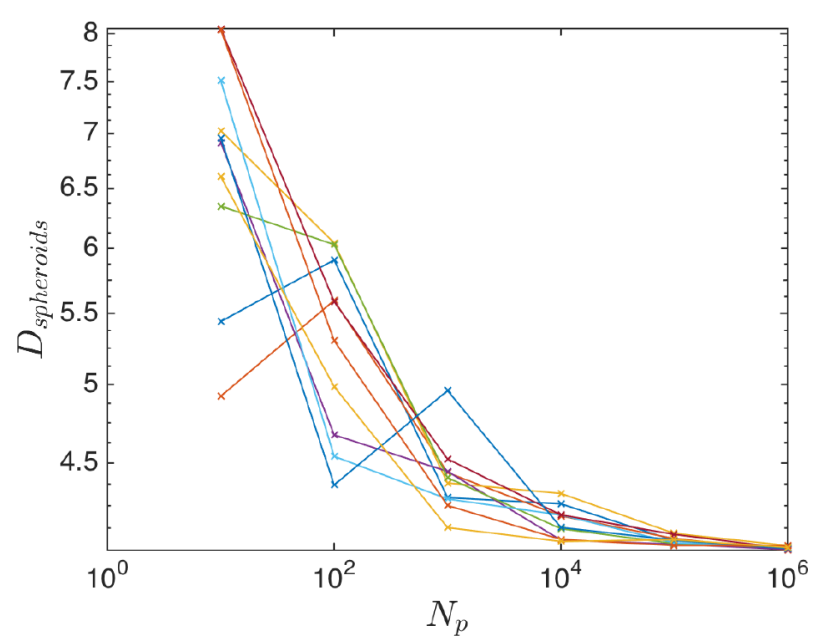

Fig. 17. Minimum of the distance, $D_{\text {spheroids }}$ between randomly generated points on the surface of two stationary spheroids as a function of the number of randomly generated points on the surface of each spheroid, $N_{p}$. The actual minimum distance between the objects is 4 lattice units and different colors represent different numerical experiments. The algorithm converges to the actual separation between the particles (4 lattice units) with an accuracy of 0.5 lattice units after $10^{4}$ iterations for all numerical experiments.
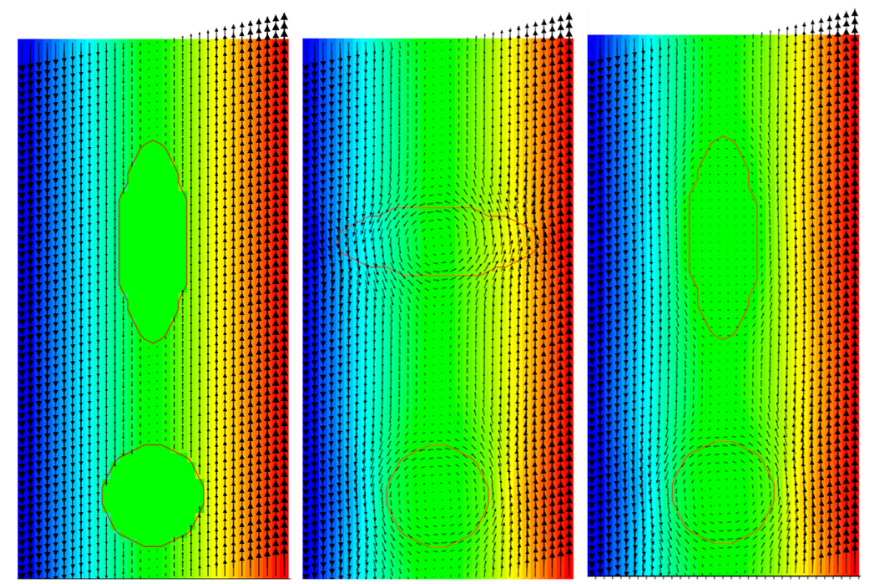

Fig. 18. A spherical particle of radius $r=6$ and a spheroid with semi-major radius $a=12$ and semi-minor radius $b=4$ placed symetrically in a simple shear flow with a separation of $30 \mathrm{LU}$ between their centers, at three different time steps, $t=0$ (left), $T / 4$ (centre) and $T / 2$ (right) where $T \approx 7200 \mathrm{LU}$ is the period of one complete rotation the spheroid.

$\left(x_{j, n}, y_{j, n}, z_{j, n}\right)$. Then the minimum distance between the surfaces, $r_{m i n, i j}$, is simply given by

$$
\min _{n=1, \ldots N p} \sqrt{\left(x_{i, n}-x_{j, n}\right)^{2}+\left(y_{i, n}-y_{j, n}\right)^{2}+\left(z_{i, n}-z_{j, n}\right)^{2}} .
$$

In order to compute the minimum of the distance of the particle from the wall, we again generate $n$ points on the surface of the particle $\mathbf{r}_{i, n}=\left(x_{i, n}, y_{i, n}, z_{i, n}\right)$. where $n$ goes from 1 to $N_{p}$. Then the minimum distance between the surfaces between the particle and the wall, $r_{m i n, i}$, is 

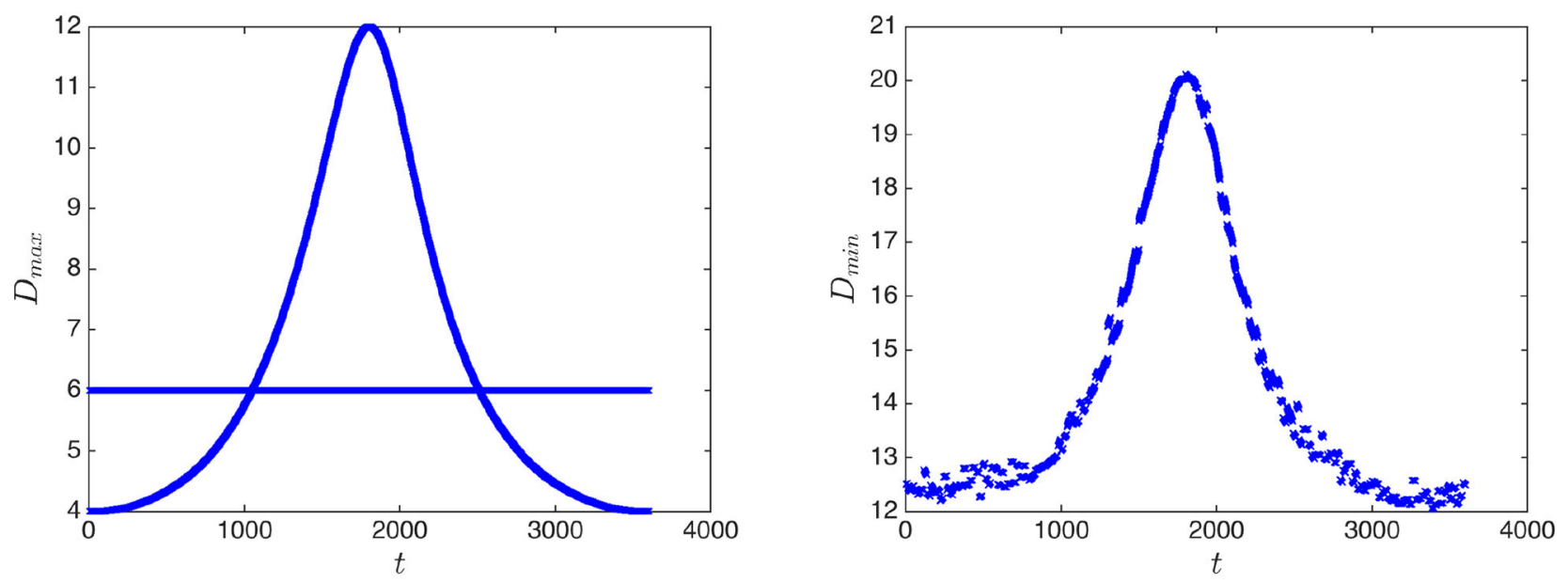

Fig. 19. Maximum distance of the particles from the center of the pipe (left) and minimum distance of the spheroid from the sphere (right).

given by

$$
r_{\min , i}=R-\max _{n=1, \ldots, N p} \sqrt{\left(y_{i, n}-y_{c}\right)^{2}+\left(z_{i, n}-z_{c}\right)^{2}},
$$

where $y_{c}$ and $z_{c}$ are coordinates of the center of pipe in non-axial direction and $R$ is the radius of the pipe. The approach is not limited to ellipsoidal geometries but is valid for particles of any arbitrary shape. Though this is a rather crude interaction model, it is expected that the total number of collisions is not sufficiently frequent to significantly alter the particle statistics in the dilute regime $(\phi<0.5 \%)$. Arcen et al. [54] showed that lubrication correction forces have a negligible effect on the statistics of spherical particles in the dilute regime. On the other hand, the more realistic approach of Lin et al. [48] is intrinsically complicated and limited to ellipsoidal geometries.

To demonstrate our approach, we compute the distance between a sphere of radius $r=6 \mathrm{LU}$ (lattice units) and a spheroid with semi-major radius $a=12 \mathrm{LU}$ and semi-minor radius $b=4 \mathrm{LU}$, placed symetrically in a simple shear flow with a separation of $30 \mathrm{LU}$ between their centers as shown in fig. 18. Figure 18 shows the flow configuration at $t=0, T / 4$ and $T / 2$ where $T \approx 7200 \mathrm{LU}$ is the period of one complete rotation of the spheroid.

As the two particles rotate due to the shear flow, the minimum distance between the surface of the two particles as well as the minimum distance of the spheroid surface from the wall changes. In order to compute the minimum distance of the spheroid surface from the wall, we randomly generate $10^{4}$ points on the surface of the spheroid and compute the maximum of its distance $\left(r_{\max }\right)$ from the center of the pipe. Then, the point of minimum separation from the wall is computed as $R-r_{\max }$, where $R$ is the radius of pipe or the half-width for the channel. As shown in fig. 19, the maximum distance of the spheroid's surface from the center of the pipe is $12 \mathrm{LU}$ and it occurs at $t=T / 4=1800$, correspondingly the minimum distance of the spheroid's surface from the wall is $R-12$ $\mathrm{LU}$ and it occurs at $t=T / 4=1800$. As expected, the maximum distance of the sphere's surface from the center of the pipe remains equal to the radius of the sphere, i.e. $6 \mathrm{LU}$ throughout the simulation. It can be seen that the method correctly predicts the minimum distance of a spheroid and sphere from the wall.

A similar process is used to compute the distance of a sphere from the spheroid and the results for both spheroid and sphere are shown in fig. 19. Here, we iteratively generate points on the surfaces of both particles and compute the minima of the distance of all randomly generated points on the surface of the spheroid to that on the sphere. The minimum distance between the two particles is $12 \mathrm{LU}$ (at $t=0$ and $t=T / 2=3600$ ) and maximum is $20 \mathrm{LU}$ at $t=T / 4=1800$. The above-discussed method very accurately predicts the distance from the wall while the distance between the particle surfaces is computed with an accuracy of $0.5 \mathrm{LU}$. In the turbulent pipe-flow simulation laden with multiple particles, we perform these iterations only when the gap between an imaginary sphere with the same radius as the major axis of the spheroid and the wall, or between the two imaginary spheres with the same radius as the major axis of the spheroid is less than one lattice unit. The random points on the surface of the particles are generated in angular coordinates using the parametric equation of the ellipsoids. They are transformed to Cartesian coordinates where the distances are computed. The total number of points generated on the surface of each particle is $10^{4}$. The surface area for S2 and S3 is 450 and $493 \mathrm{LU}^{2}$ respectively. This gives the number of points per unit surface area as 22.2 and 23.9, respectively, for the S2 and S3. The random points numbers were generated using the $\operatorname{rand}()$ function in $\mathrm{C}$, while $\operatorname{srand}()$ function sets the starting point(seed) for the random number generation. Overall, the method results in a numerical overhead of about $10-20 \%$ in our simulations, depending on the aspect ratio and the number of particles.

Open Access This is an open access article distributed under the terms of the Creative Commons Attribution License (http://creativecommons.org/licenses/by/4.0), which permits unrestricted use, distribution, and reproduction in any medium, provided the original work is properly cited. 


\section{References}

1. J.J. Stickel, R.L. Powell, Annu. Rev. Fluid Mech. 37, 129 (2005).

2. E. Molina, J.M. Fernandez-Sevilla, G. Acien Microalgae, mass culture methods, in Encyclopedia of Industrial Biotechnol.: Bioprocess, Bioseparation, and Cell Technology (John Wiley \& Sons, 2010) pp. 1-24.

3. F. Toschi, E. Bodenschatz, Annu. Rev. Fluid Mech. 41, 375 (2009).

4. Mehdi Niazi Ardekani, Léa Al Asmar, Francesco Picano, Luca Brandt, Int. J. Heat Fluid Flow 71, 189 (2018).

5. S. Balachandar, J.K. Eaton, Annu. Rev. Fluid Mech. 42, 111 (2010).

6. F. Picano, G. Sardina, C.M. Casciola, Phys. Fluids 21, 093305 (2009).

7. H. Gao, H. Li, L.P. Wang, Comput. Math. Appl. 65, 194 (2013).

8. F. Picano, W.P. Breugem, L. Brandt, J. Fluid Mech. 764, $463(2015)$.

9. A. TenCate, J.J. Derksen, L.M. Portela, H.E.A. Van Den Akker, J. Fluid Mech. 519, 233 (2004).

10. P. Costa, F. Picano, L. Brandt, W.P. Breugem, Phys. Rev. Lett. 117, 134501 (2016).

11. I. Lashgari, F. Picano, W.P. Breugem, L. Brandt, Phys. Rev. Lett. 113, 254505 (2014).

12. A. Gupta, H.J.H. Clercx, F. Toschi, Commun. Comput. Phys. 23, 665 (2018).

13. A. Gupta, H.J.H. Clercx, F. Toschi, Eur. Phys. J. E 41, 34 (2018).

14. G.A. Voth, A. Soldati, Annu. Rev. Fluid Mech. 49, 249 (2017).

15. G.B. Jeffery, Proc. R. Soc. London A: Math. Phys. Eng. Sci. 102, 161 (1922).

16. H. Brenner, Chem. Eng. Sci. 18, 1 (1963).

17. J. Happel, H. Brenner, Low Reynolds Number Hydrodynamics: With Special Applications to Particulate Media. Mechanics of Fluids and Transport Processes (Noordhoff International Publishing, 1973).

18. M. Do-Quang, G. Amberg, G. Brethouwer, A.V. Johansson, Phys. Rev. E 89, 013006 (2014).

19. N.M. Ardekani, P. Costa, W.P. Breugem, F. Picano, L. Brandt, J. Fluid Mech. 816, 43 (2017)

20. A. Eshghinejadfard, A. Abdelsamie, S.A. Hosseini, D. Thevenin, AIP Adv. 7, 095007 (2017).

21. J.P. Matas, J.F. Morris, E. Guazzelli, Phys. Rev. Lett. 90, 014501 (2003).

22. P. Patro, S.K. Dash, J. Fluids Eng. 136, 011301 (2013).

23. J.D. Kulick, J.R. Fessler, J.K. Eaton, J. Fluid Mech. 277, 109 (1994).

24. J.L. Lumley., Annu. Rev. Fluid Mech. 1, 367 (1969).

25. J.S. Paschkewitz, Y. Dubief, C.D. Dimitropoulos, E.S.G. Shaqfeh, P. Moin, J. Fluid Mech. 518, 281 (2004).

26. J.J.J. Gillissen, B.J. Boersma, P.H. Mortensen, H.I. Andersson, J. Fluid Mech. 602, 209 (2008).

27. P.K. Ptasinski, B.J. Boersma, F.T.M. Nieuwstadt, M.A Hulsen, B.H.A.A. van Den Brule, J.C.R. Hunt, J. Fluid Mech. 490, 251 (2003).
28. Y. Dubief, C.M. White, V.E. Terrapon, E.S.G. Shaqfeh, P. Moin, S.K. Lele, J. Fluid Mech. 514, 271 (2004).

29. F.T.M. Nieuwstadt, J.M.J. den Toonder, Drag Reduction by Additives: A Review (Springer Vienna, 2001) pp. 269316 .

30. L.H. Zhao, H.I. Andersson, J.J.J. Gillissen, Phys. Fluids 22, 081702 (2010).

31. G. Bellani, M.L. Byron, A.G. Collignon, C.R. Meyer, E.A. Variano, J. Fluid Mech. 712, 41 (2012).

32. S.L. Ceccio, Annu. Rev. Fluid Mech. 42, 183 (2010).

33. R.A. Verschoof, R.C.A. van der Veen, C. Sun, D. Lohse, Phys. Rev. Lett. 117, 104502 (2016).

34. J. Lin, W. Zhang, Z. Yu, J. Aerosol Sci. 35, 63 (2004).

35. S.S. Dearing, M. Campolo, A. Capone, A. Soldati, Exp. Fluids 54, 1419 (2012).

36. O. Bernstein, M. Shapiro, J. Aerosol Sci. 25, 113 (1994).

37. H. Zhang, G. Ahmadi, F.G. Fan, J.B. McLaughlin, Int. J. Multiphase Flow 27, 971 (2001).

38. P.H. Mortensen, H.I. Andersson, J.J.J. Gillissen, B.J. Boersma, Phys. Fluids 20, 093302 (2008).

39. C. Marchiolli, M. Fantoni, A. Soldati, Phys. Fluids 22 , 033301 (2010).

40. N.R. Challabotla, L. Zhao, H.I. Andersson, J. Fluid Mech. 766, R2 (2015).

41. N.R. Challabotla, Z. Lihao, H.I. Andersson, Phys. Fluids 27, 061703 (2015)

42. L. Zhao, N.R. Challabotla, H.I. Andersson, E.A. Variano, Phys. Rev. Lett. 115, 244501 (2015).

43. J. Lin, X. Shi, Z. Yu, Int. J. Multiphase Flow 29, 1355 (2003).

44. S. Succi, The Lattice Boltzmann Equation for Fluid Dynamics and Beyond. Numerical Mathematics and Scientific Computation, 1st edition (Oxford University Press, 2001).

45. Z. Guo, C. Shu, Lattice Boltzmann Method and Its Applications in Engineering (World Scientific Publishing Company, 2013).

46. Y. Chen, Q. Cai, Z. Xia, M. Wang, S. Chen, Phys. Rev. E 88, 013303 (2013).

47. M.P. Allen, D.J. Tildesley, Computer Simulation of Liquids (Clarendon Press, New York, NY, USA, 1989).

48. L. Anhua, P.H. Shih, SIAM J. Optim. 13, 298 (2002).

49. F. Lucci, A. Ferrante, S. Elghobashi, J. Fluid Mech. 650, 5 (2010).

50. S.B. Pope, Turbulent Flows (Cambridge University Press, 2000).

51. C. Alessandro, M. Massimo, P.R. Giovanni, Int. J. Multiphase Flow 94, 189 (2017) (Supplement C).

52. A. Eshghinejadfard, A. Abdelsamie, S.A. Hosseini, D. Thevenin, Int. J. Multiphase Flow 96, 161 (2017) (Supplement C).

53. S. Parsa, E. Calzavarini, F. Toschi, G.A. Voth, Phys. Rev. Lett. 109, 134501 (2012).

54. B. Arcen, A. Taniare, B. Oesteria, Int. J. Multiphase Flow 32, 1326 (2006). 